Cette version est très proche de la version publiée. Citer comme « Metzger, P., Rebotier, J. (2021). L'accouchement difficile de la société du risque et la relégation des sciences sociales. Dans Rebotier, J. (dir.) Les risques et l'anthropocène. Regards alternatifs sur l'urgence environnementale. ISTE, Londres, pp. 151-173 ».

Chapitre 6

\title{
L'accouchement difficile de la société du risque et la relégation des sciences sociales
}

\subsection{Introduction}

La société du risque ${ }^{1}$ fait référence à une lecture de l'évolution du monde social à travers le prisme du risque. Malgré l'ancienneté de la question des risques dans

Chapitre rédigé par Pascale MEtzger et Julien Rebotier. 
l'interprétation du monde social et des modes de gouverner (Ewald et Kessler 2000), un nouveau grand récit se déploie, celui de l'avènement d'une modernité réflexive qui prend au sérieux les effets dommageables de la science et de la technologie, et qui débouche sur de nouvelles formes du politique. Cependant, on constate une certaine incapacité des sciences sociales à formuler les défis posés par les risques et l'environnement en termes politiques. Les cadrages de la notion d'anthropocène contribuent à renforcer une vision naturaliste et technique qui évacue ou marginalise les approches sociales et politiques (voir le Chapitres 8). C'est tout le paradoxe de la société du risque qui d'un côté identifie les mutations de la modernité et ses implications à travers les risques, sans faire sérieusement progresser la reconnaissance des dimensions sociales et politiques des questions de risques, celles-ci restant principalement rabattues sur le physique.

En partant d'une réflexion sur la société du risque, ce chapitre vise à mettre en lumière la marginalisation objective des sciences sociales et leurs difficultés à penser le risque de façon automne. Le foisonnement d'articles et d'ouvrages scientifiques sur les risques fait écho à la prolifération des risques qui semble contaminer le monde social (Bourg et al. 2013). Inséré dans une nébuleuse de positions contradictoires, le terme risque apparait simultanément comme l'objet de trois mondes distincts: social, matériel et scientifique (Metzger 2017). Une discussion critique mais peu audible de l'anthropocène (voir Introduction Générale) s'efface derrière un cadrage naturaliste qui place au cœur des questions de risque et d'environnement objets matériels et phénomènes physiques. Les sciences sociales considèrent les choses physiques et matérielles du point de vue des constructions sociales, politiques et techniques. Elles manient des concepts flous, instables et toujours rediscutés (Becerra 2012), ce qui concourt systématiquement à mettre à distance leurs problématiques au détriment des approches de sciences dures, plus légitimes. C'est là un premier obstacle épistémologique, qui tient aux objets, aux problématiques et aux méthodes des différentes disciplines. A cela s'ajoute le système de contraintes et les rapports de domination en vigueur au sein du champ scientifique, posant frontalement le problème de l'autonomie des sciences sociales. La production des recherches en sciences sociales est toujours plus contrainte par la commande institutionnelle qui oriente et finance les recherches, en plus d'exiger une recherche pluri ou interdisciplinaire. Ce qui se diffuse de la notion d'anthropocène est ancré dans les sciences dures et tend à renforcer cette tendance. Dans ce cadre, une production significative des sciences sociales implique une forme de rébellion

${ }^{1}$ L'emploi de société du risque sans italique renvoie au moment historique de sociétés contemporaines aux prises avec les risques, qui fait notamment l'objet de la Société du risque, en italique, essai de Beck (2001). 
face à la demande sociale et à l'interdisciplinarité dominée par les sciences dures pour résister à la relégation.

Pour comprendre la position difficile des sciences sociales autour des risques et de l'environnement en ces temps d'anthropocène, on dépliera dans un premier temps les ambigüités de la Société du risque (Beck 2001), vue comme une mise en risque d'un monde engagé sur la voie de la modernité réflexive. La partie suivante explicitera les apports des sciences sociales qui à la fois confirment et critiquent les thèses de la Société du risque. La dernière partie portera sur les conditions sociales de production de la recherche et les difficultés institutionnelles que rencontrent les sciences sociales pour penser le risque de façon autonome et pour que leur voix compte.

\subsection{La Société du risque, un grand récit ambigu}

L'ouvrage de Beck (1986, traduit en français en 2001) est une référence incontournable dans la recherche sur les risques, mais aussi sur la modernité, la mondialisation ou le cosmopolitisme. Or sans définition du risque, le propos est d'emblée placé à distance de la démarche académique (Chauvel et Ramaux 2002). Essai de théorie sociale prospective plus que démonstration scientifique, son ambigüité méthodologique (Pierret 2008) rend d'autant plus paradoxal son statut de référence scientifique. Le texte fait cependant écho aux préoccupations environnementales qui agitent la sphère scientifique, le monde social et l'espace public mondial, et ouvre de nombreuses pistes de réflexion.

\subsubsection{Un essai prospectif de théorie sociale}

L'auteur part du déclin de la société industrielle moderne marqué par la prolifération des risques, la fin des grands récits structurants et de l'idéologie du progrès, et la recomposition des grandes institutions sociales (famille, État, travail). Par une analyse de l'évolution de ces structures sociales, de l'individualisation et des nouvelles formes de mobilisation, Beck nous met sur la «voie d'une autre modernité », réflexive : la société du risque en voie d'émergence.

\subsubsection{Prolifération et reconfiguration des risques}

Beck part du constat que les sociétés industrielles modernes sont confrontées à des risques toujours plus importants, toujours moins maitrisables, certains irréversibles, souvent invisibles. Le propos est illustré par les menaces globales et environnementales, les dangers technologiques, les catastrophes nucléaires et les 
controverses de santé publique qui ont marqué les années 1970-1980 (Bhopal, Seveso, OGM, sang contaminé, marées noires, pluies acides, etc.). La prolifération des risques issus de la modernité industrielle du XIXème siècle empêche d'imputer les dangers à des causes externes. Les risques ne sont plus dans la nature mais dans le développement. La production de richesses est systématiquement corrélée à la production sociale de risques.

Les risques sont globaux, «abolissent les frontières », «débordent les États nations ». Ils sont souvent invisibles, dans le sens où leur mise en visibilité est médiatisée par la science et la technique, via un arsenal de mesures et d'expertises, qui leur permettent d'« accéder à l'existence scientifique » (Beck 2001, p. 62). Ces risques s'introduisent non seulement au cœur du monde social et politique, mais aussi dans la vie quotidienne et l'intimité des personnes, en devenant « les passagers aveugles de la consommation normale» (Beck 2001, p. 17).

Face à ces risques qui génèrent peurs et angoisses dans toute la société, selon Beck, impossible de lutter. D'une part, les politiques de prévention reposent sur des experts et des scientifiques contestés, dont les connaissances sont avant tout disciplinaires, nécessairement limitées et incertaines. D'autre part, il n'est plus possible de se protéger contre les risques globaux devenus non maitrisables dans les cadres politiques nationaux. Enfin les tentatives de maîtrise du risque engendrent à leur tour de nouveaux risques.

\subsubsection{Les sciences et techniques en question}

L'analyse du rôle de la science et des savoirs est considérée comme un apport majeur de la Société du risque (Bonneuil 2002). Beck met l'accent sur une rupture dans la modernité. Alors qu'elle était portée par les valeurs centrales de progrès et de rationalité incarnées par la science et la technique, il apparaît que la modernité ne produit pas seulement des richesses mais aussi des risques toujours plus incontrôlables. Avancées scientifiques et technologiques produisent toutes sortes de menaces et de dangers, directs et induits, visibles et invisibles, dont la portée fait aujourd'hui directement écho à l'anthropocène. Pour autant, les promesses de développement (fin de la pauvreté, des inégalités, des famines, etc.) ne sont pas tenues (Dubar 2002).

Les conséquences destructrices de la modernisation, notamment dans le domaine sanitaire, deviennent des affaires publiques. La controverse s'empare de toute la société pour contester la légitimité et l'autorité des savoirs et des institutions démocratiques de régulation (Bonneuil 2002). Dans ces conditions, la société «ne peut plus se moderniser en s'appuyant sur la rationalité et le progrès, productrices de 
risques, elle ne doit plus déléguer la mise en œuvre de cette modernisation aux chercheurs, aux industriels et aux financiers » (Dubar 2002).

Aujourd'hui, le statut de la science est ambigu à plusieurs titres. Si l'exercice de la science joue un rôle crucial dans la production des risques, on attend aussi d'elle qu'elle invente des solutions, dans le contexte paradoxal d'une remise en cause de la « religion moderne du progrès ».

\subsubsection{Disqualification et recompositions du politique}

Face à la puissante alliance entre science, technique et capitalisme, Beck constate une diminution progressive du rôle du politique, voire même sa paralysie. Les choix politiques et les options futures sont moins du ressort du parlement ou des partis politiques que des laboratoires de recherche et des conseils d'administration (Beck 2001, p. 472). Les risques sont «produits par l'industrie, externalisés sur le plan économique, individualisés sur le plan juridique, légitimés sur le plan scientifique et minimisés sur le plan politique » (Vandenberghe 2001). Science et technique jouent un rôle décisif dans le recul du politique car sous couvert de neutralité et d'objectivité, technocratie et rationalité portent un projet politique invisible. Les institutions politiques ne font plus qu'administrer une évolution non maitrisée de la société tandis que les décisions se prennent ailleurs (Murard 2002), selon les principes supérieurs de rationalité, d'efficacité, de profit.

Face à la puissance de risques globaux, omniprésents, éminemment destructeurs et produits de chaines de causalités complexes (Pierret 2008), c'est l'Étatprovidence qui est remis en cause. Sont également ébranlés le calcul probabiliste et la gestion assurantielle des risques sur lesquels il repose (Ewald et Kessler 2000 ; Castel 2009), contribuant encore plus à décrédibiliser le politique par la dilution des responsabilités. Parallèlement, de nouveaux produits apparaissent sur le marché via des solutions assurantielles adaptées aux situations inédites d'incertitude croissante. Une nouvelle configuration du politique émerge alors progressivement. La «société réflexive » est celle qui pourra mettre fin « au monopole de la connaissance par les scientifiques et les experts » et à «l'opacité des processus de décision », comme le mentionne la Société du risque de Beck qui fait entrer la science en politique.

\subsubsection{Restructuration du social}

Mais le projet de Beck intervient dans un monde social transformé par une individualisation grandissante (Murard 2002) qui accroît incertitudes et risques en déstructurant institutions et formes sociales issues de la modernité industrielle. C'est le cas pour la famille, les rapports de genre et le marché du travail, alors qu'apparaissent des liens et des rôles sociaux inédits (Dubar 2002). 
En parallèle de la dépolitisation du politique dissimulée dans et par la rationalité technique, de nouvelles formes de mobilisation apparaissent autour de la prise de conscience des risques. Des initiatives de base (mouvements écologistes, participation citoyenne, associations de la société civile) se constituent hors du jeu formel de la politique et ne doivent plus rien aux catégories de la société industrielle (Pierret 2008). Les risques donnent naissance à des «communautés de menaces objectives » transversales aux catégories sociales, qui évacuent l'évidence d'une exposition et de vulnérabilités socialement différentiées.

Entre la globalisation des risques, la disqualification des classes sociales et des identités nationales, la fin du rôle de l'État et des structures sociales traditionnelles, le politique capté par le secteur privé et la science, c'est le panorama d'une forme de cataclysme social dont il question.

\subsubsection{Les ambigüités de la Société du risque...}

La prophétie politique de Beck comme l'analyse sur laquelle elle s'appuie sont loin de faire l'unanimité (Chauvel et Ramaux 2002 ; Vandenberghe 2001; Murard 2002). Malgré des apports majeurs à la compréhension du monde contemporain, le propos véhiculé par la Société du risque apparaît paradoxal et libéral.

\subsubsection{1. ... éminemment paradoxale}

Beck pointe une forme de dépolitisation des risques, évidente dans la critique de l'expertise (Henry et al. 2015 ; Boudia et Demortain 2014 ; Reghezza 2015), qu'il s'agit de repolitiser par l'avènement de la société du risque. Or pour répondre aux risques, on assiste à une demande de plus de science qui produit plus de risques, intensifiant l'appel à plus de science. La critique de la science et du progrès portée par la réflexivité devient le support d'une forme "d'expansion de la science » (Bonneuil 2002). Si la science produit des risques en les dévoilant, elle contribue aussi par des "corrections du risque » à l'apparition de nouveaux risques, dans d'autres champs, d'autres lieux ou d'autres temps (Metzger et D'Ercole 2011). Dans cette optique, plus de science entraine plus de risques qui entrainent plus de science et non pas plus de politique, alors même que la science est une forme d'exercice du politique.

L'autre grand paradoxe est la critique des logiques du capitalisme portée par la Société du risque, en même temps que l'affirmation de son expansion inéluctable. Le risque et la sécurité constituant un horizon infini de marché (Bonneuil 2002), le capitalisme se coule dans les habits du développement durable et de la gestion des risques. Partant de ce constat, Beck postule un mouvement général et irréversible de 
la globalisation technique et économique, celui « d'un ralliement à l'inévitabilité du capitalisme» (Vibert 2012). Les structures de classes s'effacent alors dans une société emportée par ce mouvement inévitable, où le moteur de l'Histoire devient la peur. Les conflits autour du partage des richesses font place aux conflits autour de la gestion et de la répartition des risques (Dubar 2002). L'enjeu n'est plus de produire la sécurité, mais d'ajuster l'état d'insécurité, de vivre -inégalement- avec le risque. Cette forme d'abdication devant l'inéluctable rappelle les critiques adressées à la notion de résilience (Quenault 2013 ; Rufat 2011 ; Reghezza 2015) et à un agenda politique libéral dépourvu de discernement social. Or si la recherche du profit et de la combinaison la plus avantageuse entre capital et travail reste le moteur d'une compétition mondialisée qui assujettit l'État (Beck 2001), les logiques du capitalisme s'attaquent aux droits sociaux historiquement acquis (Castel 2009).

\subsubsection{2. ... fondamentalement libérale}

En prônant l'émergence des individus et en soulignant l'incapacité des États, la Société $d u$ risque est indiscutablement libérale. Dans cette société sans classe et universelle, l'émergence d'un sujet réflexif en vient à disqualifier les pensées collectives du social. Plus que la fin du capitalisme, la Société du risque annonce sans détour la «liquidation péremptoire du prolétariat comme sujet politique » (Murard 2002), un discours typique de la sociologie libérale sur la fin des classes sociales (enrichissement partagé, accélération de la mobilité, dissolution des référents culturels, amoindrissement de la conscience sociale et politique - Chauvel et Ramaux 2002). Beck identifie un capitalisme qui conserve ses structures et ses problèmes d'inégalités sociales en même temps que l'apparition d'individus libérés des structures sociales et confrontés à un avenir commun (Vandenberghe 2001). Sans classe sociale, ni citoyenneté démocratique, ni régulation par l'État qui n'a plus le contrôle, l'individualisation devient le seul horizon (Chauvel et Ramaux 2002). Dans ce cadre, le politique est incarné par de nouvelles formes d'actions collectives qui ne doivent plus rien aux institutions sociales héritées. Selon Beck, la société du risque aboutit à l'émergence d'un sujet réflexif et autonome. L'individu devient une forme de parangon des idées libérales, évacuant les pensées du social qui s’appuient sur des collectifs, des institutions et des régulations socialement construites.

L'essai de Beck prend acte du fait que les États entérinent les mécanismes du marché comme forme dominante de la régulation du social. L'impuissance des États à traiter les enjeux environnementaux est vérifiée par les échecs des négociations mondiales, la puissance des acteurs économiques de la mondialisation, la poursuite de la course au progrès et aux innovations, la permanence des activités productrices de risques. Au sein même des nations, l'État-providence recule, incapable de résister à la mondialisation. Les réformes se multiplient, qui tendent toutes à diminuer la couverture des risques sociaux (santé, retraite, chômage) et à réduire le champ des 
services publics (Ewald et Kessler 2000 ; Castel 2009). La désocialisation de l'État provient de sa soumission à l'économie capitaliste et aux arguments de la rationalité scientifique qui légitiment l'action publique. Le gouvernement technocratique met en péril l'État social comme la démocratie (Vandenberghe 2001) et fonde une rhétorique qui postule l'absence d'alternative dans le contexte de la mondialisation néolibérale : there is no alternative.

\subsubsection{Une pensée prospective à actualiser}

Près de 40 ans après sa publication, à l'heure du changement climatique, des préoccupations environnementales et du succès de la collapsologie, on peut se demander ce qu'il en est de la société du risque entendue comme l'avènement d'une société réflexive qui prend au sérieux les risques qu'elle produit, et qui renouvelle la démocratie mise à mal par la technocratie.

En premier lieu, on acte le fait que le risque est un point d'entrée pertinent pour comprendre le monde social. En ces temps d'anthropocène, la mise sur agenda généralisée des questions de risque est caractéristique des sociétés contemporaines. Or on constate l'intensification d'une gestion du monde à la fois libérale et technocratique. La multiplication de mouvements sociaux simultanément locaux et mondiaux ne se traduit pas par un approfondissement démocratique de la gestion des risques, qui reste formulée comme une question avant tout technique et scientifique (voir Chapitre 5). L'ouverture à d'autres types de connaissances relève de la rhétorique puisque ces derniers ne remettent pas en cause l'autorité du discours scientifique, mais assoient au contraire sa légitimité et en relaient la diffusion dans le monde social.

Par ailleurs, la Société du risque renvoie plutôt à une société égalitaire, impliquant des « communautés de menaces objectives ». Ce point de vue n'est pas sans rappeler une idée naïve de l'anthropocène selon laquelle «on » (les humains) est «tous dans le même bateau». Or, si certaines menaces sont effectivement susceptibles de toucher «tout le monde », les formes d'expositions, les moyens de s'en protéger, d'y faire face ou de vivre avec, sont loin d'être les mêmes pour tous. Les risques conservent leur caractère discriminant. Outre l'ultime valeur qu'est la liberté et les demandes pressantes de sécurité, la notion cardinale de justice reconnait les différentiations irréductibles du monde (Smith 2010). Le monde de l'Anthropocène n'y échappe pas, en contradiction totale avec la vision égalitariste de Beck. 


\subsection{Des apports contrastés des sciences sociales}

La science et la technologie productrices de risque et de modernité, auxquelles la Société $d u$ risque fait référence, semblent ne renvoyer qu'au champ des sciences dures. On peut alors s'interroger sur la contribution des sciences sociales à la problématique des risques comme à la connaissance du monde social. Les analyses des sciences sociales confirment la thèse de la Société du risque en même temps qu'elles la complexifient, la contestent, voire la contredisent.

\subsubsection{L'évolution du rapport au risque}

\subsubsection{De l'illusion de la maîtrise à la reconnaissance de l'incertitude}

En France, la problématique du risque fait son entrée dans les sciences sociales à partir des années 1980, par les catastrophes et la notion de « risque technologique majeur » formalisée dans la Civilisation du risque (Lagadec 1981). Cet ouvrage est basé sur l'analyse d'évènements catastrophiques des années 1960-70 sous l'angle de la vulnérabilité des systèmes techniques. On en retrouve l'argumentaire dans la Société du risque: nouveauté des dangers causés par des choix technologiques et organisationnels, impacts incontrôlables dans l'espace et dans le temps, incapacité des mesures de sécurité à maîtriser le danger, conséquences économiques, politiques et sociétales, remise en question de la démocratie, appelant un sursaut de responsabilité collective et l'ouverture des prises de décisions technologiques aux citoyens.

Un second ouvrage marquant est La société vulnérable (Theys et Fabiani 1987) qui traite conjointement des risques et de l'environnement du point de vue de la recherche et de la gestion. Il y est pointé que la vulnérabilité de la société reflète l'urbanisation, la concentration des activités et le développement de technologies toujours plus complexes et dangereuses, mais aussi l'amélioration des performances des systèmes techniques et une sécurité croissante.

Vingt-cinq ans après La société vulnérable, l'ouvrage Du risque à la menace (Bourg et al. 2013) conteste l'idée d'une société réflexive, soucieuse de limiter les effets secondaires et dangereux des développements technoscientifiques : dans la mesure où «le risque et ses modalités de contrôle sont devenus producteurs de menace » la recherche et l'action accompagnent les dangers plus qu'ils ne les limitent. L'ouvrage s'interroge sur ce que les approches en termes de risque entendu comme un danger pris en charge, calculable et maitrisable- rendent visible ou dissimulent. Désormais, plus que le risque, la « menace » englobe tout un registre de phénomènes depuis l'évènement «naturel» exceptionnel jusqu'aux menaces 
économiques ou politiques, susceptibles de s'enchainer de façon incontrôlable, renvoyant à l'idée de « risque majeur » (Lagadec 1981). La menace -vue comme un ensemble de dangers imbriqués impossibles à maitriser- n'entre plus dans la " mise en risque » (Ewald 1986) qui inscrit les dangers dans des procédures de gestion. Elle échappe aux formes de maîtrise et de réduction de l'incertitude propres au risque.

Ce glissement des approches par les sciences sociales du risque à la menace traduit une montée des incertitudes et renvoie à ce qui peut caractériser l'anthropocène (Reghezza 2015). C'est dans ce cadre qu'on assiste à la substitution de la vulnérabilité par la résilience.

\subsubsection{De la Vulnérabilité à Résilience}

Terme clé des sciences sociales, la vulnérabilité renvoie initialement à une certaine fragilité et à une susceptibilité d'endommagement, celles-ci résultant de l'exposition à des aléas comme de conditions sociales, économiques, politiques, techniques (Quenault et al. 2011; Becerra 2012). Ultérieurement, la notion de vulnérabilité s'est étendue aux capacités des groupes sociaux face aux risques. $\mathrm{La}$ vulnérabilité n'est plus la conséquence du risque sur un monde social passif mais l'aptitude du monde social à influer sur le risque. La vulnérabilité s'incarne concrètement dans les conséquences différenciées de l'impact d'un aléa (extérieur au monde social) en fonction des individus, des groupes sociaux, des sociétés, ou des territoires (Blaikie et al. 1994).

Cependant, la vulnérabilité est presque toujours posée face à un aléa -vu comme phénomène extérieur au monde social. Cette idée d'extériorité de la menace est en contradiction avec la Société du risque. Une rare définition qui ne fait pas référence à l'aléa est la suivante: "social vulnerability derives from the activities and circumstances of everyday life or its transformation $»^{2}$ (Hewitt 1997, cité par Quenault et al. 2011). Cette formulation permet de penser une vulnérabilité ancrée dans le fonctionnement normal du monde social, affranchie de l'aléa et de la catastrophe. Une rupture inédite s'opère dans la vision de la vulnérabilité mais aussi du risque lui-même.

Depuis les années 2000, le terme « résilience » s'est propagé dans les sciences sociales et les politiques publiques, comme un écho à la Société $d u$ risque libérale et individualiste de Beck. La résilience exprime la capacité à absorber des chocs et à retrouver un fonctionnement normal après une perturbation (Quenault 2013), de quelque nature qu'elle soit. Dans le domaine des risques, la résilience en vient à

2 «La vulnérabilité sociale est issue des activités et des conditions de la vie quotidienne ou de son évolution » $=>$ Garder l'original pour la version en anglais. 
disputer la centralité de la vulnérabilité pour s'ériger en solution universelle. Malgré les nombreuses critiques dont elle fait l'objet (Rufat 2011; Bourcart 2015), la résilience s'impose en évoquant la possibilité d'une rupture épistémologique avec les visions technicistes du risque et de l'environnement (Djament-Tran et ReghezzaZitt 2012).

La substitution de la résilience, qui fait écho à la vision libérale du monde, à la vulnérabilité, porteuse d'une critique du développement, est un asservissement des modes de penser des sciences sociales. La résilience participe d'une forme d'acceptation des risques et des crises pour lesquels le rôle des acteurs politiques devient secondaire (Quenault 2015 ; Petit et al. 2014). La responsabilité de faire face aux crises est redistribuée parmi les individus dont les conduites, scrutées, servent des techniques de gouvernement (Borraz 2013; Felli 2014). Sous couvert d'une critique convenue des approches technocratiques et descendantes, de la promotion de l'autonomie des communautés et des individus, de leurs capacités propres à « faire face » aux risques et aux crises, à «s'éduquer» et à «s'adapter » aux « nouvelles menaces» (Revet 2011), la résilience dédouane États et pouvoirs publics de leur rôle moderne dans l'organisation du monde social (voir Chapitre 4).

La diffusion large de la résilience comme solution universelle acte l'échec des critiques de la notion, incapables de faire exister une pensée du risque et de sa gestion qui n'évacue pas le projet politique. La science politique analyse le risque comme « technique de gouvernement [...] qui contribue à reconfigurer le social et le politique » (Borraz 2013). Le risque est ainsi ancré dans le champ du social et du politique, résultat d'un travail de construction. En cela, les recherches en sciences sociales documentent des processus de construction des risques et d'organisation du social dans lesquels science et technologie comptent mais parmi bien d'autres éléments. Avec quels résultats ?

\subsubsection{Des apports incontestables mais peu audibles autour du risque}

\subsubsection{Trois acceptions de la construction sociale}

Si l'idée de «construction sociale » du risque fédère les approches du risque par les sciences sociales, il convient d'en identifier au moins trois conceptions. La première renvoie à la production «concrète» de risques par la société et le (mal)développement (Beck 2001; Theys et Fabiani 1987). Une conception radicalement opposée considère la «mise en risque » ou la «fabrique des risques » (Ewald 1986; Gilbert 2003), en référence à tous les processus sociaux, investissements, alignements d'acteurs qui vont aboutir à la formulation d'un problème en termes de risque calculable qui peut être géré dans le cadre de 
politiques publiques (Gilbert et Henry 2009). Cette conception fait référence à quelque chose de tout aussi réel, qui se joue à l'intérieur du monde social, à propos de choses qui engagent du danger matériel.

Tout autre est le fait de dire que le risque «n'existe pas» (Kermisch 2012). Derrière cette formulation abrupte, on trouve d'une part l'idée que le risque est toujours potentiel, probable et calculé, et à ce titre n'existe pas. Quand le risque «se réalise », quand il devient «réel», il s'appelle «accident», «crise» ou « catastrophe ». On trouve d'autre part les approches par les représentations ou les perceptions qui questionnent la réalité objective du risque en mobilisant des démarches à la fois sociologiques, psychologiques et anthropologiques (PerettiWatel 2000). Le risque est vu comme une croyance, une perception, une représentation dans la lignée d'analyses culturalistes et behavioristes. S'il est subjectif, sa réalité et sa condition objective sont évacuées. Les sciences dures sont particulièrement réceptives aux analyses de perception des risques qui renforcent leur position : les perceptions sociales n'ayant pas de réalité matérielle et objective, elles n'existent pas. La seule réalité légitime du risque est fondée sur l'approche physique des phénomènes dommageables, à l'aune de quoi toute mesure est prise.

\subsubsection{Le statut de l'évènement : un défi épistémologique}

La plupart des travaux sur le risque prennent directement ou indirectement la catastrophe comme point de départ ou comme perspective, voire les deux. En tant qu'événement, la catastrophe, passée ou probable, est au cœur du risque. On peut la situer dans l'ordre de l'extraordinaire qui délimite un avant et un après : " le risque et sa réalisation renvoient ainsi derechef à un phénomène exceptionnel » (Bourg et al. 2013). Or, "pour les sciences sociales, l'évènement pose problème » (Bensa et Fassin 2002) dans la mesure où leur ambition est de dégager des formes de «lois » et de « régularités » du social... que l'évènement semble suspendre (Morin 1972). D'où la nécessité épistémologique pour les sciences sociales de repositionner l'évènement dans des régularités du social. Ceci se fait d'une part par des analyses qui dégagent des régularités dans le déroulement des crises (Lagadec 1981 ; Godard et al. 2002), d'autre part par des efforts de réinscription du risque et des crises dans la normalité des processus politiques et sociaux (Gilbert et Lascoumes 2003 ; Ewald 1986 ; Hewitt 1997 ; Gilbert 2013).

Ces approches posent le risque comme une forme de normalité, inscrite dans les modalités mêmes du fonctionnement et du développement de la société moderne (Beck 2001 ; Borraz 2013). Dans une vision du risque tiraillée entre l'exceptionnel et l'ordinaire, l'événement est de plus en plus disqualifié. S'intéresser à « ce qui fait tenir » le monde au quotidien, en cohérence avec une société qui produit sans cesse de nouveaux risques, ouvre des perspectives de recherches: penser la sécurité 
ordinaire loin des situations de crise (Gilbert 2016). A ce titre, «l'événement» anthropocène interroge s'il ne permet pas d'expliciter la mécanique ordinaire d'un monde social aux prises avec des enjeux environnementaux inédits.

Qu'il s'agisse de risques naturels, technologiques, ou environnementaux, les risques sont rendus visibles par la science qui désigne certains objets ou phénomènes (amiante, OGM, pluies acides) comme dangereux à travers des dispositifs de mesure techniques et normatifs. Dans ce cas c'est la science -ici les sciences dures- qui désigne des dangers potentiels. Mais pour les sciences sociales, désigner le risque implique de passer par le monde social et les controverses tenues dans des arènes élargies (bien au-delà du périmètre des sciences dures). C'est la fabrique des risques qui inscrit les problèmes dans le champ des risques (Ewald 1986).

Cependant, la question du risque soulève une tension entre ignorance et connaissance propre au champ scientifique : «loin de faire reculer l'ignorance, le savoir accroît l'incertitude et par là même le risque » (Luhman cité par Le Bouter 2014 ; Henry et al. 2015). Cette position renvoie à la science post-normale (Ravetz 1992) selon laquelle la science ne fait pas reculer l'ignorance, mais accroît l'inconnu et l'incertain. Les tensions grandissantes entre incertitude et connaissance au cœur des questions de risque expliquent le développement de travaux sur l'expertise et les rapports entre sciences et société (Henry et al. 2015). Un tel développement ouvre la voie à la sociologie des sciences, dans la mesure où la question des risques implique nécessairement controverses et scepticisme (Larrère et Larrère 2013; Gilbert et Henry 2009). La simple question de savoir s'il y a ou non danger est objet de controverses : le vrai n'est plus décidable par la seule connaissance scientifique qui ne jouit plus de l'argument d'autorité. On assiste alors à une forme d'ouverture de la décision au monde social par des processus de reconnaissance de savoirs profanes, de participation ou de conférences de consensus, qui constituent autant de nouveaux objets de recherche pour les sciences sociales. C'est tout l'intérêt d'une interprétation en termes de « mise en risque » et de « fabrique des risques » (Gilbert 2003) qui pose la résolution de la controverse, toujours provisoire, non pas dans le champ de la science normale (controverses métrologiques), mais dans celui, contingent, du monde social (controverses axiologiques).

Les sciences sociales informent sur ce qui fait risque, à quel titre, au terme de quel processus. Or l'analyse des conditions de production de la connaissance sur les risques lève également le voile sur les positions relatives dans lesquelles sont tenues les sciences sociales au sein du champ scientifique. 


\subsection{Les sciences sociales prises dans un univers de contraintes}

Force est de constater que les connaissances apportées par les sciences sociales ont un impact très limité sur le monde social et la formulation des politiques publiques. Les sciences sociales pâtissent d'un manque de légitimité sur les problématiques de risques et d'environnement, notamment face aux sciences dures. Elles subissent également des conditions sociales de production qui accroissent leur position de subordination au sein du champ scientifique (Bourdieu 1975).

\subsubsection{La supériorité du calcul sur le discours}

On repère dans le monde social et scientifique une croyance en la capacité supérieure des sciences dures à dire le vrai, appuyé par des chiffres, des mesures, et des dispositifs techniques. Pour la «raison technique moderne» (Feenberg 2002), calcul et rationalité, considérés comme vrais, neutres, objectifs, et universels, fondent un principe supérieur de légitimation (Boltanski et Thévenot 1991; Godard 2004)

Malgré les développements sur la perte de confiance dans la science et la technique, la rhétorique du chiffre reste centrale pour la démonstration et l'évaluation (Godard 2004), comme en atteste la diffusion tous azimuts du new public management, du pilotage par les indicateurs ou du benchmarking qui mesurent l'efficacité des individus et des organisations. Il en va de même pour l'évolution des conditions de production de la recherche scientifique (classement de Shanghai, scores de publication -Bouleau 2003, p. 88), alors que «toute quantification est une extension possible du marché ». En cela, la science -dure- et la technique détiennent les instruments de légitimation des sociétés capitalistes avancées.

On est bien là dans le registre de la modernité industrielle, où le calcul participe à la désignation du risque. Pour les experts comme pour les pouvoirs publics, le risque demeure objectivable par une fonction entre probabilité d'occurrence d'un phénomène et quantité de dommages qu'il pourrait provoquer. C'est l'énoncé classique "aléa x vulnérabilité » que l'on retrouve aussi bien dans la recherche qu'au cœur des politiques de prévention. Or les chiffres dissimulent aussi l'ignorance et conduisent à une réduction factice de l'incertitude. Pourtant, la suprématie de la quantification et des dispositifs instrumentaux qui sont l'apanage des sciences dures et de la technologie continue d'opérer.

Par contraste, les connaissances apportées par les sciences sociales sont souvent de l'ordre du discours interprétatif. Elles situent et expliquent leur objet de recherche 
dans leur contexte historique, social, politique et territorial. Au motif d'un relativisme excessif ou d'un constructionnisme radical, les sciences sociales sont rapidement taxées de contingentes, subjectives, voire idéologiques et dogmatiques, surtout quand elles portent sur la mise au jour de rapports de domination (voir les critiques faites à Bourdieu). A l'inverse, elles sont plutôt bien acceptées quand elles produisent du chiffre, alors même que le recours à la quantification opère une mise en équivalence des objets, une disqualification de l'interprétation, une éviction du sens (Lemoine 2009 ; Bouleau 2013).

Depuis une quinzaine d'années, les appels à projets insistent systématiquement sur la pluridisciplinarité et la nécessité de prendre en compte les dimensions sociales du risque. Mais ces dernières consistent le plus souvent en une vision très limitée de la vulnérabilité (comptage d'éléments exposés à un aléa, perception des risques). De tels attendus, tant de la part des sciences dures que des gestionnaires, posent le risque en termes de sensibilisation et d'acceptation, et permettent d'œuvrer à la mise en place des politiques publiques, loin de l'explicitation de la fabrique des risques.

\subsubsection{Conditions financières et orientations problématiques}

De façon générale, la recherche est toujours plus dépendante de financements sur projets, en réponse à une demande institutionnelle. En France, l'Agence Nationale de la Recherche sert la «mise en œuvre du financement de la recherche sur projets », sur un mode de «sélection compétitive, qui respecte les standards internationaux ». Son objectif est certes, de «contribuer au développement des sciences et des technologies » et de « favoriser la créativité », mais aussi de « cibler les efforts de recherche sur des priorités économiques et sociétales définies au plus haut niveau de l'État», «encourager les interactions entre disciplines » et « intensifier les liens public-privé $»^{3}$. L'orientation explicite de la recherche scientifique est dictée par les objectifs et priorités de l'État, dans un contexte concurrentiel et pluridisciplinaire (Metzger 2017). En ce sens, on assiste à une perte d'autonomie du champ scientifique dans sa globalité (Bourdieu 2001), perte plus accusée encore pour les sciences sociales car l'autonomie repose aussi sur un « droit d'entrée ». Il s'agit de la compétence incorporée par la discipline pour le droit à dire le vrai. Or « tout le monde se sent en droit de se mêler de sociologie et d'entrer dans la lutte à propos de la vision légitime du monde social » (Bourdieu 2001). Il n’en va pas de même dès lors qu'il est question de modélisation du climat.

\footnotetext{
${ }^{3}$ http://www.agence-nationale-recherche.fr/missions-et-organisation/missions/
} 
La pluridisciplinarité ne remet pas en cause les rapports de domination existants au sein du monde scientifique. Sur les questions de risque et d'environnement, les sciences dures sont considérées comme les véritables détentrices de la connaissance, laissant aux sciences sociales le rôle de danseuse (Pestre 2004). La hiérarchie des disciplines est inscrite dans le fonctionnement institutionnel de la recherche. Elle se traduit par un engagement asymétrique des sciences dures et des sciences sociales dans les projets pluridisciplinaires sur les risques et l'environnement, et par un formatage de la demande sociale qui impose une vision naturaliste des problématiques (Metzger 2017).

La demande institutionnelle en termes de recherche porte d'abord sur la connaissance des phénomènes physiques impliqués dans les risques, donc sur une connaissance apportée (et une recherche pilotée) par les sciences dures (Metzger 2017). En conséquence, les sciences sociales doivent se plier au cadrage conceptuel et problématique dominant. Elles sont assignées à produire des connaissances dans les «espaces à risques » ou sur les "populations vulnérables » désignés par les sciences dures ou utiles à la mise en place des politiques publiques. Elles sont poussées vers une forme d'ingénierie sociale consacrée à l'évaluation comptable de la vulnérabilité, de la résilience, des perceptions du risque et de l'acceptabilité peu compatible avec l'analyse de la fabrique des risques.

\subsubsection{Entre attente d'opérationnalité et critique inaudible}

L'opérationnalité de la recherche en réponse à la demande sociale contribue à sa légitimité. Or attendre des sciences sociales qu'elles servent à quelque chose, «c'est toujours leur demander de servir le pouvoir, alors que [leur] fonction scientifique est de comprendre le monde social, à commencer par les pouvoirs» (Lahire 2004). La démarche en sciences sociales procède d'abord d'une reformulation des « problèmes » énoncés par la demande sociale (Castel 2009), ce qui n'arrange pas des «demandeurs » qui s'exposent à recevoir des réponses à une question qu'ils n'ont pas posée.

Si la pertinence des discours sur la dimension sociale des risques est bien établie, seuls certains aspects en sont effectivement mobilisés. Les gestionnaires du risque sont demandeurs d'une évaluation des « éléments exposés 》 et de la « vulnérabilité » de la population qui permet des représentations chiffrées et opérationnelles sous forme de cartes et d'indicateurs. L'évaluation comptable de la vulnérabilité s'appuie sur des caractéristiques socio-économiques (sur lesquelles les politiques de prévention n'agissent généralement pas), ou documente perceptions sociales et comportements de la population identifiés comme problématiques du fait des distorsions avec le « vrai » risque dit par les sciences dures (c'est-à-dire l'aléa). 
Le point de vue qui consiste à poser la population comme incompétente, irrationnelle et ignorante sur les questions de risque (Joly 2001) exclut de prendre au sérieux les savoirs profanes de la population. Dans le même temps, la diversité des risques auxquels la population doit faire face mais aussi l'arbitrage nécessaire entre intérêts contradictoires encapsulés dans les politiques de gestion sont négligés (Dourlens 1991; Metzger et D’Ercole 2011). Focalisées sur ce que dit et fait la population «face aux risques », ces pratiques des sciences sociales participent à la diffusion du point de vue des sciences dures (Peretti-Watel 2010; Coanus et al. 2010), fondant des politiques de «sensibilisation» pour développer «bonnes pratiques » et «culture du risque » (Revet 2011). Aussi, les sciences sociales sont celles qui savent «parler aux gens ». Elles sont sollicitées pour «traduire » les connaissances de l'aléa, pour mieux les faire accepter et favoriser l'adoption de bons comportements.

Mais s'en tenir à une position critique et radicale fondée sur la vulnérabilité, c'est entériner la faible opérationnalité de la notion dans la mesure où l'action concrète de réduction des risques devient impossible (Metzger et Robert 2015). Face à l'opérationnalité toujours plus attendue, les approches critiques de la vulnérabilité sont incapables de proposer des transformations des modalités du développement que les politiques de prévention pourraient mettre en œuvre. L'insertion formelle de la vulnérabilité dans les politiques publiques a neutralisé sa portée critique en l'enfermant dans une vision comptable des pertes potentielles, ou dans un apport utilitariste sur les perceptions et comportements de la population.

En revanche, l'interprétation du risque comme nouveau principe de gouvernement et de structuration du monde social (Borraz 2013; Revet et Langumier 2013) est possible parce qu'il s'agit d'un éclairage qui ne revendique pas une utilité opérationnelle directe. Cette lecture du risque n'a pas vocation à diminuer les risques ni à intervenir dans des situations concrètes, mais à les expliquer, à décrypter leur rôle dans la configuration du monde social. En cela, les sciences sociales construisent une autonomie inversement proportionnelle à l'écho qu'elles ont dans l'espace public, ce que semble confirmer le moment anthropocène.

\subsubsection{L'impérieux cadrage de l'anthropocène}

Longtemps les sciences sociales ont résisté à investir l'environnement comme objet de recherche (Henry et Jollivet 1998). Arc-boutées sur les « faits sociaux », les sciences sociales se trouvent dans l'incapacité épistémologique de considérer les « faits de nature » impliqués dans la question environnementale (Boudes 2008), ce qui se traduit par une certaine faiblesse conceptuelle (dommageable en contexte d'interdisciplinarité) et par des recherches pragmatiques et finalisées. Depuis les 
années 1990, ce champ de recherche opère un renversement significatif. Alors qu'on reprochait initialement aux sciences sociales de ne travailler l'environnement que sous l'angle de sa construction sociale, il semble qu'il ne soit plus que milieu naturel (Kalaora et Vlassopoulos 2013). Difficile aujourd'hui de trouver une définition de l'environnement qui ne renvoie pas au milieu « naturel» (Metzger 2017).

Du fait d'un cadrage dominant par les sciences dures et de la portée limitée des approches critiques de sciences sociales, l'anthropocène ressort de la même tendance. Dans la vision du monde qui s'impose, les sciences dures, éminemment légitimes, jouent un rôle fondamental, malgré la reconnaissance de leurs limites et des incertitudes croissantes (réflexivité de la Société du risque). Catégorie scientifique de la géologie, l'anthropocène postule «l'humain » comme catégorie du monde social.

«L'humain », catégorie indifférenciée, renvoie à une condition biologique qui nie la question sociale. La problématique environnementale comme l'anthropocène postulent une communauté de destin qui correspond à l'échelle de la planète, de l'histoire de l'évolution ou des temps géologiques. Mais dès qu'il s'agit de situations concrètes, de diversité du monde, le nous humains relève de l'idéologie. Campée sur la légitimité d'une vérité biologique, cette idée masque les différentiations sociales qu'incarnent le risque et l'environnement, notamment à travers les intérêts contradictoires et les conflits qu'ils occasionnent. La stratégie performative du nous humains capable de faire advenir une forme de solidarité planétaire entre (tous) les hommes, (toutes) les sociétés et les mécanismes biogéochimiques de la planète ne peut faire office d'agenda intellectuel des sciences sociales. Dans un monde qui ressort plus de la compétition que de la solidarité, c'est pourtant ce nous qui s'impose, produisant des catégories de vision et de di-vision du monde social qui font éclater les anciennes catégories sociales. Ces visions, propres aussi à la Société $d u$ risque, s'ancrent dans le rapport des hommes et des sociétés aux risques (population vulnérable, humains, femmes, jeunes, etc.). C'est là une façon d'oblitérer les rôles du développement du capitalisme et de la mondialisation (Smith 2010), la violence des rapports Nord-Sud dans la période de la colonisation et de l'ère industrielle (Ferdinand 2019), la responsabilité du mode de développement néolibéral dans la «dégradation de l'environnement» (Hornborg 2017). En bref c'est éluder les systèmes d'exploitation et de domination qui ont façonné le monde social.

En produisant un discours généralisé sur le devenir «humain » et en déclinant les enjeux environnementaux impérieux, de l'individu à la planète, l'anthropocène s'institue comme paradigme scientifique qui relègue dans les mêmes limbes la connaissance critique des sciences sociales et l'action politique émancipatrice. En 
cela, l'autonomie des sciences sociales et leur aptitude à formuler les questions des risques et de l'environnement comme question sociale et politique demeurent capitales. Paradoxalement, plus les sciences sociales s'investissent, plus elles sont soumises à des objectifs opérationnels et un cadre pluridisciplinaire, plus elles ont tendance à adopter une approche naturalisée et gestionnaire de l'environnement (Castree et al. 2014).

\subsection{Conclusion}

Malgré son succès et la pertinence de nombreux points, la Société du risque n'aboutit pas à une transformation du monde par l'avènement d'une modernité réflexive. Certes, la société du risque est là. Les risques, globaux et locaux, invisibles et matérialisés, ont fait irruption dans l'espace public, autour du changement climatique, des pesticides ou des nanotechnologies. Partout dans le monde fleurissent des actions collectives de protestation ou de revendication sur ces sujets. Les négociations multilatérales sur les questions environnementales rythment l'agenda politique international, tandis que discours et recherches sur l'intégration des savoirs profanes et des contre expertises citoyennes se multiplient.

Or science, technologie ou encore innovation conservent leur centralité. Malgré la réflexivité déployée, elles continuent de fonder la croissance économique comme les solutions aux risques. Dans le champ politique, la mise en démocratie des risques n'est pas d'actualité. Les arènes de négociation dissimulent mal l'asymétrie des acteurs et la permanence de l'hégémonie de la rationalité scientifique. Dans le champ économique, la question environnementale sert l'expansion du capitalisme (marchés de droits à polluer, du vivant).

La Société du risque pose implicitement l'hypothèse que les décisions sont prises en fonction de la connaissance scientifique et de la rationalité, ce qui au mieux est naï, au pire totalement inexact. Quand la modernité réflexive parle de prendre au sérieux les effets induits de la science et la technologie, ou de démocratiser la gestion des risques, on voit arriver l'anthropocène, la résilience et le développement durable, avec leur lot d'actions inéluctables.

Face à l'urgence, quand «il n'y a pas d'alternative », la solution qui s'impose n'est pas la meilleure mais celle qui reste après qu'on s'est privé des possibilités de faire autrement. Comprendre le monde de l'anthropocène suppose d'en maîtriser le cadre d'analyse, et par conséquent de s'arracher aux conditions de production de la recherche. Cette insubordination a un coût que le fonctionnement institutionnel de la recherche rend exorbitant, si ce n'est à la marge ou loin de l'action. Mais qui a dit que l'émancipation viendrait de la connaissance scientifique? 


\subsection{Bibliographie}

Becerra, S. (2012). Vulnérabilité, risques et environnement: l'itinéraire chaotique d'un paradigme sociologique contemporain, [VertigO] La revue électronique en sciences de l'environnement, 12(1) [En ligne]. Disponible à l'adresse : https://journals.openedition.org/vertigo/11988 [Consulté le 10 mai 2020].

Beck, U. (2001). La Société du risque. Aubier, Paris.

Bensa, A., Fassin, E. (2002). Les sciences sociales face à l'événement, Terrain, 38, 5-20.

Blaikie, P., Cannon, T., Davis, I., Wisner, B. (1994). At Risk: Natural Hazards, People's Vulnerability and Disasters. Routledge, London.

Boltanski, L., Thévenot, L. (1991). De la justification: les économies de la grandeur. Gallimard, Paris.

Bonneuil, C. (2002). A plusieurs voix sur la société du risque, Mouvements, 21-22(3), 171174.

Borraz, O. (2013). Pour une sociologie critique des risques. Dans Du risque à la menace. Penser la catastrophe, Bourg et al., 237-255.

Boudes, P. (2008). L'environnement, domaine sociologique. La sociologie française au risque de l'environnement. Thèse de doctorat en sociologie, Université Bordeaux 2, Bordeaux.

Boudia, S., Demortain, D. (2014). La production d'un instrument générique de gouvernement, Gouvernement et Action Publique, 3(3), 33-53.

Bouleau, N. (2013). L'excessive mathématisation, symptômes et enjeux. Dans Du risque à la menace. Penser la catastrophe, Bourg et al., 79-105.

Bourcart, L. (2015). Emergence et usages du concept de résilience dans les mondes académique et instutionnel. Thèse de doctorat en science politique, Université Grenoble Alpes, Grenoble.

Bourdieu, P. (1975). La spécificité du champ scientifique et les conditions sociales du progrès de la raison, Sociologie et Société, 7(1), 91-118.

Bourdieu, P. (2001). Science de la science et réflexivité. Raisons d'agir, Paris.

Bourg, D., Joly, P-B., Kaufmann, A. (dir.). (2013). Du risque à la menace. PUF, Paris.

Castel, R. (2009). La montée des incertitudes. Seuil, Paris.

Castree, N., Adams, W.M., Barry, J., Brockington, D., Büscher, B., Corbera, E., Demeritt, D., Duffy, R., Felt, U., Neves, K., Newell, P., Pellizzoni, L., Rigby, K., Robbins, P., Robin, L., Rose, D.B., Ross, A., Schlosberg, D., Sörlin, S., West, P., Whitehead, M., Wynne, B. (2014). Changing the Intellectual Climate, Nature Climate Change, 4, 763 768 .

Chauvel, L., Ramaux, C. (2002). A plusieurs voix sur la société du risque, Mouvements, 2122(3), 166-170. 
Coanus, T., Comby, J., Ducheneet, F., Martinais, E. (dir.). (2010). Risques et territoires. Interroger et comprendre la dimension locale de quelques risques contemporains. Lavoisier, Paris.

Djament-Tran, G., Reghezza-Zitt, M. (dir.). (2012). Résiliences urbaines, les villes face aux catastrophes. Éditions du manuscrit, Paris.

Dourlens, C., Galand, J-P., Theys, J., Vidal-Naquet, P. (1991). Conquête de la sécurité, gestion des risques. L'Harmattan, Paris.

Dubar, C. (2002). Note de lecture, la société du Risque sur la voie d'une autre modernité, Revue française des affaires sociales, 2, 211-215.

Ewald, F. (1986). L'État providence. Grasset, Paris.

Ewald, F., Kessler, D. (2000). Les noces du risque et de la politique, Le Débat, 2(109), 55-72.

Feenberg, A., David, J. (2002). Marcuse et l'esthétisation de la technologie, Quaderni, 49, 81101.

Felli, R. (2014). Adaptation et résilience : critique de la nouvelle éthique de la politique environnementale internationale, Éthique Publique, 16(1) [En ligne]. Disponible à l'adresse: https://journals.openedition.org/ethiquepublique/1371 [Consulté le 14 mai 2020].

Ferdinand, M. (2019). Une écologie décoloniale - Penser l'écologie depuis le monde caribéen. Seuil, Paris.

Gilbert, C. (2003). La fabrique des risques, Cahiers internationaux de sociologie, 114(1), 5572 .

Gilbert, C. (2013). Quels risques pour la recherche en sciences humaines et sociales. Dans $D u$ risque à la menace. Penser la catastrophe, Bourg et al., 219-236.

Gilbert, C. (2016). Revisiter les risques à l'aune de la sécurité ordinaire. Dans Faire face aux risques dans les sociétés contemporaines, Becerra, S., Lalanne, M., Weisbein, J. (dir.). Octaresed, Toulouse, 267-274.

Gilbert, C., Henry, C. (dir.). (2009). Comment se construisent les problèmes de santé publique. La Découverte, Paris.

Gilbert, C., Lascoumes, P. (2003). Les politiques des risques en Europe, Revue internationale de politique comparée, 10(2), 151-160.

Godard, O. (2004). De la pluralité des ordres - les problèmes d'environnement et de développement durable à la lumière de la théorie de la justification, Géographie, économie, société, 3(6), 303-330.

Godard, O., Henry, C., Lagadec, P., Michel-Kerjean, E. (2002). Traité des nouveaux risques. Gallimard, Paris.

Henry, C., Jollivet, M. (1998). Introduction. Dans La question de l'environnement dans les sciences sociales. Éléments pour un bilan, PEVS du CNRS, Paris. 
Henry, E., Gilbert, C., Jouzel, J-N., Marichalar, P. (2015). Dictionnaire critique de l'expertise. Santé, travail, environnement. Presses de Sciences Po, Paris.

Hewitt, K. (1997). Regions of Risk: A Geographical Introduction to Disasters. Routlege, Londres \& New York.

Hornborg, A. (2017). Dithering while the planet burns: Anthropologist's approaches to the Anthropocene, Reviews in Anthropology, 46(2-3), 61-77.

Joly, P-B. (2001). Les OGM entre la science et le public? Quatre modèles pour la gouvernance de l'innovation et des risques, Économie rurale, 266, 11-29.

Kalaora, B., Vlassopoulos, C. (2013). Pour une sociologie de l'environnement: environnement, société et politique. Champ Vallon, Paris.

Kermisch, C. (2012). Vers une définition multidimensionnelle du risque, [VertigO] La revue électronique en sciences de l'environnement, 12(2) [En ligne]. Disponible à l'adresse : https://journals.openedition.org/vertigo/12214 [Consulté le 14 mai 2020].

Lagadec, P. (1981). La civilisation du risque. Catastrophes technologiques et responsabilité sociale. Seuil, Paris.

Lahire, B. (dir.). (2004). A quoi sert la sociologie? La Découverte, Paris.

Larrère, C., Larrère, R. (2013). Peut-on échapper au catastrophisme? Dans Du risque à la menace, Bourg et al., 199-216.

Le Bouter, F. (2014). La sociologie constructiviste du risque de Niklas Luhmann, Communication et organisation, 45, 33-48.

Lemoine, B. (2009). Compte Rendu: Alain Desrosières, l'argument statistique pour une sociologie historique de la quantification (tome 1) et gouverner par les nombres (Tome 2), Revue d'anthropologie des connaissances, 3(2), 359-365.

Metzger, P. (2017). Connaissances et relations de pouvoir : les sciences sociales, les risques et l'environnement. Habilitation à Diriger des Recherches, Université Grenoble Alpes, Grenoble.

Metzger, P., D'Ercole, R. (2011). Les risques en milieu urbain : éléments de réflexion, EchoGéo, 18

Metzger, P., Robert, J. (2015). Environnement et risques : les sciences sociales piégées entre critique radicale et utilité sociale. Dans Espaces et rapports de domination, Clerval, A., Fleury, A., Rebotier, J., Weber, S. (dir.). PUR, Rennes, 69-79.

Murard, N. (2002). A plusieurs voix sur la société du risque, Mouvements, 21-22(3), 162-165.

Pestre, D. (2004). L'évolution des champs de savoir, interdisciplinarité et noyaux durs, Natures Sciences Sociétés, 12, 191-196.

Peretti-Watel, P. (2000). Sociologie du risque. Armand Collin, Paris.

Petit, O., Hubert, B., Theys, J. (2014). Science globale et interdisciplinarité : quand contagion des concepts rime avec confusion, Natures Sciences Sociétés, 22(3), 187-188. 
Pierret, J. (2008). De la société du risque à la société réflexive. Beck peut-il sauver la modernité ? Dans Les ambivalences du risques, Cartuyvels, Y., Presses de l'Université Saint-Louis, Bruxelles, 27-63.

Quenault, B. (coord.), Bertrand, F., Blond, N., Glatron, S., Pigeon, P., Peyrache-Gadeau, V., Rocher, L. (2011). Vulnérabilité et résilience au changement climatique en milieu urbain : vers des stratégies de développement urbain durable? MSHB, Rennes.

Quenault, B. (2013). Retour critique sur la mobilisation du concept de résilience en lien avec l'adaptation des systèmes urbains au changement climatique, EchoGéo, 24 [En ligne]. Disponible à l'adresse : https://journals.openedition.org/echogeo/13403 [Consulté le 10 mai 2020].

Quenault, B. (2015). De Hyōgo à Sendai, la résilience comme impératif d'adaptation aux risques de catastrophe : nouvelle valeur universelle ou gouvernement par la catastrophe ? Développement durable et territoires, 6(3) [En ligne]. Disponible à l'adresse: https://journals.openedition.org/developpementdurable/11010 [Consulté le 16 mai 2020].

Ravetz, J. (1992). Connaissance utile, ignorance utile ? Dans La Terre outragée. Les experts sont formels. Theys, J., Kalaora, B. (dir.). Éditions Autrement, Paris, 87-101.

Reghezza, M. (2015). De l'avènement du Monde à celui de la planète : le basculement de la société du risque à la société de l'incertitude. Habilitation à Diriger les Recherches, Université Paris 1- Panthéon Sorbonne.

Revet, S. (2011). Penser et affronter les désastres : un panorama des recherches en sciences sociales et des politiques internationales, Critique internationale, 52(3), 157-173.

Revet, S., Langumier, J. (dir.). (2013). Le gouvernement des catastrophes. Karthala, Paris.

Rufat, S. (2011). Critique de la résilience pure [En ligne]. Disponible à l'adresse: https://hal.archives-ouvertes.fr/hal-00693162 [Consulté le 10 mai 2020].

Smith, N. (2010). Uneven Development: Nature, Capital, and the Production of Space, $3^{\text {rd }}$ edition. University of Georgia Press, Athens and London.

Theys, J., Fabiani, J-L. (1987). La société vulnérable : évaluer et maîtriser les risques. Presses de l'École Normale Supérieure, Paris.

Vandenberghe, F. (2001). Introduction à la sociologie (cosmo)politique du risque d'Ulrich Beck, Revue du MAUSS, 17(1), 25-39.

Vibert, S. (2012). Une démocratie sans société ? Critique de Beck et de l'idéologie cosmopolitique en sociologie, Sociologie et sociétés, 44(1), 121-140. 